\title{
EXPANSIONS OF INVERSE SEMIGROUPS
}

\author{
MARK V. LAWSON, STUART W. MARGOLIS and BENJAMIN STEINBERG ${ }^{\circledR}$
}

(Received 24 January 2002; revised 31 March 2004)

Communicated by D. Easdown

\begin{abstract}
We construct the freest idempotent-pure expansion of an inverse semigroup, generalizing an expansion of Margolis and Meakin for the group case. We also generalize the Birget-Rhodes prefix expansion to inverse semigroups with an application to partial actions of inverse semigroups. In the process of generalizing the latter expansion, we are led to a new class of idempotent-pure homomorphisms which we term $F$-morphisms. These play the same role in the theory of idempotent-pure homomorphisms that $F$-inverse monoids play in the theory of $E$-unitary inverse semigroups.
\end{abstract}

2000 Mathematics subject classification: primary 20M18, $20 \mathrm{M} 17$.

Keywords and phrases: Inverse semigroups, expansions, $F$-inverse semigroups, prehomomorphisms, Birget-Rhodes expansion.

\section{Introduction}

Expansions have played an important role in the semigroup theory literature, yet the only widely used 'expansions' in inverse semigroup theory have had as domain the category of groups: namely the prefix expansion of Birget and Rhodes [3], and the Cayley graph expansion of the second author and Meakin [11]. In this paper, we, amongst other things, make these constructions true expansions by generalizing them to arbitrary inverse semigroups. Applications are given to partial actions of inverse semigroups.

The prefix expansion turns out to have a universal property in terms of what we shall call $F$-morphisms. These are surjective homomorphisms $\varphi: S \rightarrow T$ of inverse semigroups such that $t \varphi^{-1}$ has a maximum for each $t \in T$; such morphisms are necessarily idempotent-pure. An inverse monoid is $F$-inverse if and only if its maximal group image homomorphism is an $F$-morphism. We show that $F$-morphisms

(C) 2006 Australian Mathematical Society $1446-7887 / 06 \$ \mathrm{~A} 2.00+0.00$ 
are the analog of $F$-inverse monoids when one transfers the theory of $E$-unitary inverse semigroups to surjective idempotent-pure homomorphisms. In particular, we prove that if $\varphi: S \rightarrow T$ is idempotent-pure, then $\varphi=j \beta$ with $j$ an embedding and $\beta$ an $F$-morphism. From this, one obtains in a straightforward way Billhardt's result on factoring idempotent-pure morphisms through $\lambda$-semidirect product projections.

\section{Notation}

Let $A$ be a set and $\widetilde{A}=A \cup A^{-1}$. We will denote by $\widetilde{A}^{+}\left(\widetilde{A}^{*}\right)$ the free semigroup (monoid) with involution on $A$ and by $F G(A)$ the free group on $A$. If $S$ is an $A$ generated inverse semigroup and $w \in \widetilde{A}^{+}$, then $[w]_{S}$ will denote the image of $w$ in $S$. If $S$ is an inverse semigroup, $E(S)$ will denote the idempotents of $S$. In this paper, we will only deal with inverse semigroups. We leave to the reader the straightforward adjustments needed to handle the case of inverse monoids.

Inverse semigroup presentations will be written $\operatorname{Inv}\langle A \mid R\rangle$ where $A$ is the generating set and $R$ the set of relations.

We use Inv for the category of inverse semigroups and homomorphisms, and we use $\operatorname{Inv}_{A}$ for the category of $A$-generated inverse semigroups with homomorphisms respecting the generators. Homomorphisms will also be called morphisms.

Green's relations and the natural partial order for inverse semigroups will be used throughout; see $[10,16]$.

\section{Expansions}

An expansion of inverse semigroups is a functor $F: \operatorname{Inv} \rightarrow \operatorname{Inv}$ equipped with a natural transformation $\eta: F \rightarrow 1_{\text {Inv }}$ such that each component $\eta_{S}: F(S) \rightarrow S$ is surjective; expansions were introduced by Birget and Rhodes [2]. In other words, an expansion assigns to each inverse semigroup $S$ a semigroup $F(S)$ and an onto morphism $\eta_{S}: F(S) \rightarrow S$ such that whenever $\tau: S \rightarrow T$ is a morphism, the following diagram commutes:

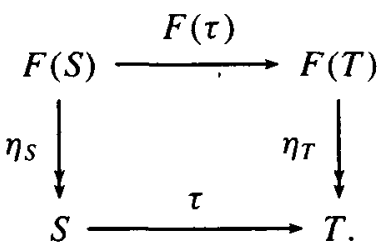

An expansion of inverse semigroups cut-to-generators is an assignment to each set $A$ of a functor $F_{A}: \mathbf{I n v}_{A} \rightarrow \mathbf{I n v}_{A}$ with a natural transformation $\eta_{A}: F_{A} \rightarrow 1_{\mathbf{I n v}_{A}}$. In general, we will consider a fixed set $A$ and drop the subscript from the notation. 


\section{Schützenberger graphs}

Recall that two elements $s, t$ of an inverse semigroup $S$ are said to be $\mathcal{R}$-equivalent if $s s^{-1}=t t^{-1}$. The $\mathcal{R}$-class of $s$ is denoted $R_{s}$. If $S$ is generated by $A$, then, for $w \in \tilde{A}^{+}$, the Schützenberger graph of $w, \mathcal{S}(w)$, has vertices $R_{[w]_{s}}$ and edges of the form $(s, a, t)$ where $a \in A, s, t \in R_{[w] s}$, and $s a=t$. If $(s, a, t)$ is an edge, then $\left(t, a^{-1}, s\right)$ will represent the same edge thought of as being traversed in the opposite direction. The idempotent $\left[w w^{-1}\right]_{S}$ is taken as the initial vertex and $[w]_{S}$ as the final vertex. Observe that $\mathcal{S}(w)$ is dependent only on $[w]_{S}$ and so we can write $\mathcal{S}(s)$, for $s \in S$, without ambiguity. Also note that any two $\mathcal{R}$-equivalent elements of $S$ have the same graph, up to the choice of the terminal vertex, while $\mathcal{D}$-equivalent elements have isomorphic Schützenberger graphs (here, up to the choice of initial and terminal vertices). Schützenberger graphs were introduced by Stephen [21].

We let $L(\mathcal{S}(w))$ be the collection of all words of $\widetilde{A}^{+}$which read a path in $\mathcal{S}(w)$ from the initial vertex to the terminal vertex. It is easy to check that $v \in L(\mathcal{S}(w))$ if and only if $[w]_{s} \leq[v]_{S}$ in the natural partial order. Schützenberger graphs are well known [21] to be inverse graphs meaning that if a Schützenberger graph has edges $(s, a, t),\left(s, a, t^{\prime}\right)$ with $a \in \widetilde{A}$, then $t=t^{\prime}$. Hence every word in $\widetilde{A}^{+}$labels at most one path into or out of any vertex. In general, an inverse automaton is a connected inverse graph with distinguished initial and terminal vertices.

We observe that if $\varphi: T \rightarrow S$ is a morphism of $A$-generated inverse semigroups respecting generators then $\varphi$ induces, for each $w \in \widetilde{A}^{+}$, an automaton morphism $\varphi_{w}: \mathcal{S}(w)_{T} \rightarrow \mathcal{S}(w)_{S}$ (where the subscript indicates which semigroup is being considered). A morphism is called idempotent-pure if the inverse images of idempotents consist of idempotents. For a morphism of $A$-generated inverse semigroups, this is equivalent to asking that $\varphi_{w}$ be an embedding for each $w \in \widetilde{A}^{+}$. This follows from the following well-known facts: a morphism is idempotent-pure if and only if it is injective when restricted to $\mathcal{R}$-classes [10]; a morphism of inverse automata is an embedding if and only if it injective on the vertex set $[20,21]$. For the case of $E$-unitary inverse semigroups, see [12].

The following key observation is due to Stephen [21].

PROPOSITION 4.1. Let $S$ be an A-generated inverse semigroup and $v, w \in \tilde{A}^{+}$. Then $[v]_{S}=[w]_{S}$ if and only if $L(\mathcal{S}(v))=L(\mathcal{S}(w))$.

In particular, if all the Schützenberger graphs of $S$ are finite, the word problem for $S$ is solvable provided the Schützenberger automata are constructible. Indeed, there is an algorithm to check whether two finite automata accept the same language. Often this can be done, see [21].

COROLLARY 4.2. Let $S$ be an A-generated inverse semigroup with finite $\mathcal{R}$-classes. Then $S$ is residually finite. 
PROOF. The transition semigroups of the Schützenberger graphs are clearly homomorphic images of $S$ and are finite since the $\mathcal{R}$-classes are finite. Suppose $v, w \in \widetilde{A}^{*}$ are such that $[v]_{S} \neq[w]_{S}$, then $L(\mathcal{S}(v)) \neq L(\mathcal{S}(w))$ by Proposition 4.1, so $v$ and $w$ cannot act the same on the vertices of both $\mathcal{S}(v)$ and $\mathcal{S}(w)$. Thus $v$ and $w$ can be separated in a finite image of $S$.

Hence, by a well-known result of Evans [6], it follows that if $S$ has finite Schützenberger graphs, is recursively presented, and one can effectively enumerate the finite inverse semigroups satisfying the defining relations of $S$, then one can decide the word problem for $S$.

The following is observed in [13].

PROPOSITION 4.3. Suppose $S=\operatorname{Inv}\langle A \mid R\rangle$ where $R$ consists of relations of the form $t^{m}=t^{m+n}$ with $n \leq m$. Then the Schützenberger graphs of $S$ are finite.

This will also follow from our results (although the proof is basically the same).

Note that if $s, t \in S$, then, since the $\mathcal{R}$-relation is a left congruence, left multiplication by $s$ on the vertex set of $\mathcal{S}(t)$ induces a labeled graph morphism from $\mathcal{S}(t)$ to $\mathcal{S}(s t)$ respecting the terminal vertices (though not necessarily the initial vertices). On the other hand, left multiplication by $s t t^{-1} s^{-1}$ is easily seen to induce a labeled graph morphism from $\mathcal{S}(s)$ to $\mathcal{S}(s t)$ respecting the initial vertices (but not necessarily the terminal vertices); this follows since $(s t)(s t)^{-1}=s t t^{-1} s^{-1} \leq s s^{-1}$. If $X$ is a subgraph of $\mathcal{S}(t)$, then $s X$ will denote the image of $X$ (in $\mathcal{S}(s t)$ ) under the action of $s$. Observe that if $t \in s^{-1} s S$ (or, equivalently, $t t^{-1} \leq s^{-1} s$ ), then left multiplication by $s$ induces an isomorphism of labeled graphs (with inverse, left multiplication by $s^{-1}$ ).

LEMMA 4.4. Let $S$ be an A-generated inverse semigroup and $u, v \in \widetilde{A}^{+}$. Suppose that $P_{u}$ is the path read in $\mathcal{S}(u)$ from $\left[u u^{-1}\right]_{S}$ by $u$ and $P_{v}$ is the path read in $\mathcal{S}(v)$ from $\left[v v^{-1}\right]_{S}$ by $v$. Then the path read by $u v$ in $\mathcal{S}(u v)$ from $\left[u v(u v)^{-1}\right]_{S}$ is $\left[u v v^{-1} u^{-1}\right]_{S} P_{u} \cup[u]_{S} P_{v}$.

PROOF. From what we have so far observed, $\left[u v v^{-1} u^{-1}\right]_{S} P_{u}$ is a path labeled by $u$ from $\left[u v(u v)^{-1}\right]_{S}$ in $\mathcal{S}(u v)$ whose endpoint must be $\left[u v v^{-1} u^{-1} u\right]_{S}=\left[u v v^{-1}\right]_{s}$. On the other hand, $u P_{v}$ is a path labeled by $v$ in $\mathcal{S}(u v)$ with endpoint $[u v]_{s}$ and hence whose starting point is $\left[u v v^{-1}\right]_{s}$. It thus follows that the path read by $u v$ in $\mathcal{S}(u v)$ is precisely $\left[u v v^{-1} u^{-1}\right]_{S} P_{u} \cup[u]_{S} P_{v}$.

We end this section with a related proposition.

LEMMA 4.5. Let $S$ be an A-generated inverse semigroup and $u \in \widetilde{A}^{+}$. Suppose $u=v w$. Then in $\mathcal{S}(u)$, the paths $u$ and $u w^{-1}$ use the same edges. Also, $u \mathcal{R} u w^{-1}$. 
PROOF. Clearly any edges used in reading $u$ is used in reading $u w^{-1}$. While reading $u=v w$ from $\left[u u^{-1}\right]_{S}$ to $[u]_{S}$, we read a path $P$ labeled by $w$ from $\left[u u^{-1} v\right]_{S}$ to $[u]_{s}$. Hence $u w^{-1}$ first traverses the path labeled by $u$ and then backtracks over the subpath $P$ : no new edges are used. The second statement follows since

$$
u w^{-1}\left(u w^{-1}\right)^{-1}=u w^{-1} w u^{-1}=v w w^{-1} w w^{-1} v^{-1}=v w w^{-1} v^{-1}=u u^{-1} .
$$

\section{Free idempotent-pure expansions}

In this section, we introduce an expansion cut-to-generators (_) ${ }^{\mathrm{Sl}}$ such that each component of the natural transformation $\eta$ is idempotent-pure, and with the following universal property: if $\varphi: T \rightarrow S$ is an idempotent-pure morphism ( $T$ generated by $A$ ), then there is a unique (necessarily idempotent-pure) morphism $\psi: S^{\mathrm{St}} \rightarrow T$ such that $\psi \varphi=\eta_{s}$.

5.1. Presentations Before giving an explicit construction, we will give a presentation for $S^{\mathrm{SI}}$ and show, using Proposition 4.3, that the Schützenberger graphs of this semigroup are finite.

Let $S=\operatorname{Inv}\langle A \mid R\rangle$. Then $S^{S 1}$ has presentation $\operatorname{Inv}\left\langle A \mid R^{\prime}\right\rangle$ where $R^{\prime}$ consists of all relations $u=u^{2}$ with $[u]_{S}$ an idempotent; if $\rho$ is the congruence associated to $S$, then the congruence associated to $S^{\mathrm{S1}}$ is denoted $\rho^{\mathrm{min}}$ in [16]. Clearly, the congruence generated by $R^{\prime}$ is contained in that of $R$, so $\eta_{S}: S^{S I} \rightarrow S$ exists. Since the image under a morphism of an idempotent is an idempotent, it is clear that $\left({ }_{-}\right)^{\mathrm{SI}}$ is a functor and $\eta$ a natural transformation. The finiteness of the Schützenberger graphs now follows from Proposition 4.3. By construction, $\eta_{S}$ is idempotent-pure and universal for this property. Note that if $S$ has decidable word problem, the above presentation is recursive.

We would like to obtain an explicit representation of this expansion (this was done for the case that $S$ is a group by the second author and Meakin [11]). This will allow us to better understand Green's relations for the resulting inverse semigroup $S^{\mathrm{Sl}}$ as well as to see such properties as, for instance, the finiteness of $S$ and $A$ implies the finiteness of $S^{\mathrm{S}}$. This can be proved by Brown's theorem [5], but our representation will make this, as well as the finiteness of the Schützenberger graphs in general, evident.

First we describe explicitly the congruence on $\widetilde{A}^{+}$giving rise to $S^{\text {sl }}$.

THEOREM 5.1. Let $S$ be an A-generated inverse semigroup. Define a relation on $\tilde{A}^{+}$by $u \equiv v$ if $[u]_{S}=[v]_{s}$, and $u$ and $v$ use the same edges in their respective runs from $\left[u u^{-1}\right]_{s}=\left[v v^{-1}\right]_{S}$ in $\mathcal{S}(u)=\mathcal{S}(v)$. Then $\equiv$ is a congruence and $S^{\mathrm{Sl}}=\widetilde{A}^{+} \equiv$.

PROOF. Clearly $\equiv$ is an equivalence relation. That it is a congruence containing the Wagner congruence [10] follows immediately from Lemma 4.4. We now show 
that it has the universal property of $S^{\mathrm{SI}}$. Suppose $\sim$ is a congruence on $\widetilde{A}^{+}$such that

$$
\begin{aligned}
u \sim v & \Longrightarrow[u]_{s}=[v]_{s}, \\
{[u]_{s}=[u]_{S}^{2} } & \Longrightarrow u \sim u^{2} .
\end{aligned}
$$

Let $T=\widetilde{A}^{+} / \sim$. Then the natural morphism $\varphi: T \rightarrow S$ is idempotent-pure. Suppose $u, v \in \widetilde{A}^{+}$and $u \equiv v$. Then we have the following: $\varphi_{u}: \mathcal{S}(u)_{T} \rightarrow \mathcal{S}(u)_{S}$ is an embedding, $u$ runs from $\left[u u^{-1}\right]_{T}$ to $[u]_{T}$ in $S(u)_{T}$, and $v$ runs from $\left[u u^{-1}\right]_{S}$ to $[u]_{S}$ in $\mathcal{S}(u)_{S}$ using edges in $\mathcal{S}(u)_{T} \varphi_{u}$. Thus $v \in L\left(\mathcal{S}(u)_{T}\right)$ whence $[u]_{T} \leq[v]_{T}$. A symmetric argument shows that $[v]_{T} \leq[u]_{T}$ so $[u]_{T}=[v]_{T}$. Therefore $\equiv \subseteq \sim$ and the result follows.

Note that if $S$ has a decidable word problem, this presentation is recursive.

COROLLARY 5.2. Let $S$ be an A-generated inverse semigroup and $u \in \widetilde{A}^{+}$such that

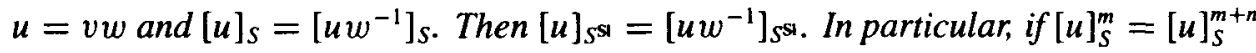
with $n \leq m$, then $[u]_{S^{\mathrm{si}}}^{m}=[u]_{S^{\mathrm{sl}}}^{m+n}$.

PROOF. This follows immediately from Lemma 4.5 .

COROLLARY 5.3. Let $S=\operatorname{Inv}\langle A \mid R\rangle$ where $R$ consists entirely of relations of the form $u=u w^{-1}$ where $u=v w, w \in \widetilde{A}^{+}$. Then $S^{\mathrm{Sl}}=S$, that is, $S$ has no non-trivial, idempotent-pure A-generated extensions. In particular, this holds if $R$ consists entirely of relations of the form $t^{m}=t^{m+n}$ with $n \leq m$.

5.2. Structure We now describe the structure of this expansion. Let $S$ be an $A$-generated inverse semigroup. Let $\mathcal{T}$ be the collection of inverse automata over $A$ which can be obtained as the (finite, connected) subautomaton of $\mathcal{S}(w)$ used in reading $w$ from $\left[w w^{-1}\right]_{S}$ to $[w]_{S}$ for some $w \in \tilde{A}^{+}$. Let $T$ be the set of all $(X, s) \in \mathcal{T} \times S$ such that $X$ has initial state $s s^{-1}$ and final state $s$ (so, by definition of $\mathcal{T}, X \subseteq \mathcal{S}(s)$ ). We define a multiplication by

$$
\left(X_{1}, s_{1}\right)\left(X_{2}, s_{2}\right)=\left(s_{1} s_{2} s_{2}^{-1} s_{1}^{-1} X_{1} \cup s_{1} X_{2}, s_{1} s_{2}\right) .
$$

From Lemma 4.4, it is clear that if $X_{1} \in \mathcal{T}$ corresponds to $w_{1} \in \widetilde{A}^{+}$and $X_{2}$ corresponds to $w_{2}$, then $s_{1} s_{2} s_{2}^{-1} s_{1}^{-1} X_{1} \cup s_{1} X_{2}$ corresponds to $w_{1} w_{2}$. Thus the multiplication on $T$ is well defined. It is easily checked to be associative and the resulting semigroup is inverse: $(X, s)^{-1}=\left(s^{-1} X, s^{-1}\right)$. In fact, the above argument shows that the map $\varphi: \widetilde{A}^{+} \rightarrow T$ defined by $w \varphi=\left(X,[w]_{S}\right)$, where $X$ is the collection of edges used in reading $w$ from $\left[w w^{-1}\right]_{S}$ to $[w]_{S}$ in $\mathcal{S}(w)$, is a surjection and that the congruence associated to $\varphi$ is $\equiv$. Thus $T=S^{\mathrm{Sl}}$. The map to $S$ is just the projection to the second coordinate. Summing it all up, we have the following result. 
THEOREM 5.4. Let $S$ be an A-generated inverse semigroup. Then $S^{S 1}$ consists of all pairs $(X, s)$ where $s \in S$ and there is a word $w \in \widetilde{A}^{+}$such that $[w]_{s}=s$ and $X$ is the subautomaton of $\mathcal{S}(w)$ used in reading $w$ from the initial vertex. The operations are given by:

$$
\begin{aligned}
\left(X_{1}, s_{1}\right)\left(X_{2}, s_{2}\right) & =\left(s_{1} s_{2} s_{2}^{-1} s_{1}^{-1} X_{1} \cup s_{1} X_{2}, s_{1} s_{2}\right), \\
(X, s)^{-1} & =\left(s^{-1} X, s^{-1}\right) .
\end{aligned}
$$

The natural transformation $\eta_{S}$ is the projection to $S$; the map from $\widetilde{A}^{+}$to $S^{\mathrm{Sl}}$ takes $w$ to $\left(X,[w]_{S}\right)$ where $X$ is the set of edges read by $w$ in $\mathcal{S}(w)$.

For example, if $S$ is a group, then $S^{S 1}$ consists of all pairs $(X, s)$ where $s \in S$ and $X$ is a finite, connected subgraph of the Cayley graph of $S$ containing 1 and $s$, so $S^{\text {st }}$ is just the expansion of [11] applied to $S$.

We observe that the above inverse semigroup does not in general consist of all pairs $(X, s)$ where $X$ is a finite, connected subgraph of $\mathcal{S}(s)$ containing $s s^{-1}$ and $s$. Indeed, if $S$ is the free inverse semigroup on $x$, then the pair $\left(X, x^{2} x^{-2}\right)$ where $X$ consists just of the edge $\left(x^{2} x^{-2}, x, x^{2} x^{-2} x\right)$ (and its reverse) is not a valid element of $S^{S I}=S$ as no word mapping to $x^{2} x^{-2}$ can avoid passing through the vertex $x^{2}$.

In [13], it is shown that for the free inverse semigroups satisfying $x^{n}=x^{n+1}$, any finite connected inverse automaton over the same alphabet whose transition inverse monoid satisfies $x^{n}=x^{n+1}$ can be a Schützenberger graph. The question for the free inverse semigroups satisfying $x^{n}=x^{2 n}$ seems interesting and hard.

We now restate Theorem 5.4 in a way which makes it clearer which graphs come up, giving a more semantic description of $S^{\mathrm{sl}}$.

PROPOSITION 5.5. Let $S$ be an A-generated inverse semigroup. Then $S^{\mathrm{Sl}}$ consists of all pairs $(X, s)$ where $X$ is a finite connected subgraph of $\mathcal{S}(s)$ containing $s s^{-1}, s$ and there is a word $w$ reading from $s^{-1}$ to $s$ using all the edges of $X$ such that $w$ cannot be read in $\mathcal{S}(t)$ (from $t t^{-1}$ to $t$ ) for any $t>s$. The operations are given by:

$$
\begin{aligned}
\left(X_{1}, s_{1}\right)\left(X_{2}, s_{2}\right) & =\left(s_{1} s_{2} s_{2}^{-1} s_{1}^{-1} X_{1} \cup s_{1} X_{2}, s_{1} s_{2}\right), \\
(X, s)^{-1} & =\left(s^{-1} X, s^{-1}\right) .
\end{aligned}
$$

The natural transformation $\eta_{S}$ is the projection to $S$; the map from $\widetilde{A}^{+}$to $S^{\mathrm{Si}}$ takes $w$ to $\left(X,[w]_{S}\right)$ where $X$ is the set of edges read by $w$ in $\mathcal{S}(w)$.

Proof. First we show that if $(X, s)$ is as in the theorem statement, then there is a word $w$ with $[w]_{s}=s$ and such that $w$ uses precisely the edges of $X$ in $\mathcal{S}(w)$ when reading from $s s^{-1}$ to $s$. Indeed, let $w$ be a word reading in $X$ from $s s^{-1}$ to $s$ using every edge of $X$ and which cannot be read on any $\mathcal{S}(t)$ (from $t t^{-1}$ to $t$ ) with $t>s$. Then $[w]_{s} \geq s$. Since $w$ can be read on $\mathcal{S}(w)$, it follows $[w]_{S}=s$. 
Suppose now that $(X, s)$ is a valid element of $S^{\text {st }}$ according to Theorem 5.4. Choose $w$ so that $[w]_{S}=s$ and $w$ uses precisely the edges of $X$ in $\mathcal{S}(w)$ when reading from $s s^{-1}$ to $s$. Suppose there exists $t>s$ such that $w$ can be read on $\mathcal{S}(t)$ from $t t^{-1}$ to $t$. Then $s=[w]_{s} \geq t$, a contradiction.

In our earlier example, any word readable on $X$ is readable on $\mathcal{S}(x)$.

In fact it is not hard to show that if $S$ is $E$-unitary, then the pairs $(X, s)$ which arise in $S^{\mathrm{Sl}}$ correspond to those subgraphs $X$ of $\mathcal{S}(s)$ containing $s s^{-1}, s$ such that there is no subgraph $Y \subseteq \mathcal{S}(t)$ with $s s^{-1} Y=X$ and $t>s$. We do not have as satisfactory a description in general.

Observe that since $\eta_{S}$ is idempotent-pure, the idempotents of $S^{\mathrm{St}}$ are all pairs of the form $(X, e)$ with $e \in E(S)$. Note that the initial and final states of $X$ will coincide.

Our coordinatization makes the following result obvious.

COROLlARY 5.6. Let $A$ be finite and $S$ be a finite A-generated inverse semigroup. Then $S^{\mathrm{SI}}$ is also finite.

Let $\mathcal{S}=\bigcup_{s \in S} \mathcal{S}(s)$. Then the left representation of $S$ on itself induces an action of $S$ on the free semilattices $F$ on the set of edges of $\mathcal{S}$. One can then realize $S^{\text {si }}$ as an inverse subsemigroup of the $\lambda$-semidirect product $F x^{\lambda} S$ in an obvious way. See $[1,10]$ for the definitions of $\lambda$-semidirect products.

Our next goal is to characterize Green's relations on $S^{\text {st }}$. In particular, we show that the Schützenberger graphs of $S^{\mathrm{Sl}}$ are finite. For the rest of this section, we fix an $A$-generated inverse semigroup $S$. Since most of the results are straightforward calculations (or one can use general results about $\lambda$-semidirect products or $L$-representations $[1,10])$, we omit the proofs.

Proposition 5.7. Let $(X, s),(Y, t) \in S^{S \mathrm{I}}$. Then $(X, s) \leq(Y, t)$ if and only if $s \leq t$ and $s s^{-1} Y \subseteq X$.

Now we can turn to the Green's relations. The easiest to handle will be the $\mathcal{R}$ relation. First we remark that it is easy to see that if $(X, s) \in S^{\text {St }}$ and $t \in \operatorname{Ver}(X)$ (where $\operatorname{Ver}(X)$ is the set of vertices of $X$ ), then $(X, t) \in S^{\mathrm{St}}$.

Proposition 5.8. Let $(X, s) \in S^{\mathrm{SI}}$. Then $(Y, t) \mathcal{R}(X, s)$ if and only if $t \mathcal{R} s$ and $X$ and $Y$ agree except for the terminal vertex.

Dually,

Proposition 5.9. Let $(X, s) \in S^{\mathrm{St}}$. Then $(Y, t) \mathcal{L}(X, s)$ if and only if $t \mathcal{L} s$ and $s^{-1} X$ and $t^{-1} Y$ agree except for the terminal vertex. 
The above results shows that the $\mathcal{L}$ and $\mathcal{R}$-classes are finite. However, much more can be said.

Proposition 5.10. Let $w \in \tilde{A}^{+}$. Then $\left(\eta_{s}\right)_{w}: \mathcal{S}(w)_{S^{s}} \rightarrow \mathcal{S}(w)_{S}$ is an embedding with image $X$ where $\left(X,[w]_{S}\right)=[w]_{S s}$. In other words, $\mathcal{S}((X, s))$ is isomorphic to $X$ under the map which forgets the first coordinate.

Proof. The two statements are clearly equivalent. By Proposition 5.8, the $\mathcal{R}$-class of an element $(X, s)$ consists of all pairs $(Y, t)$ where $Y$ is obtained by changing the terminal vertex of $X$ to $t$. Thus $\eta_{s}$ maps the vertices of $\mathcal{S}((X, s))$ bijectively to the vertices of $X$. To complete the proof, we need to show that if $(s, a, t)$ is an edge of $X$, then $((X, s), a,(X, t))$ is an edge of $\mathcal{S}((X, s))$. But we have already seen $(X, s) \mathcal{R}(X, t)$ and if $w \in \widetilde{A}$ maps to $(X, s)$, then since $(s, a, t)$ is an edge of $X \subseteq \mathcal{S}(t)$, it follows that $[w a]_{S}=t$ and uses precisely the edges of $X$. Thus $((X, s), a,(X, t))$ is an edge of $\mathcal{S}((X, s))$ as desired. The result follows.

COROLLARY 5.11. For any set $A$ and A-generated inverse semigroup $S$, the Schützenberger graphs of $S^{\mathrm{SI}}$ are all finite.

The $\mathcal{H}$-relation has a particularly pleasant description.

PROPOSITION 5.12. Let $(X, e)$ be an idempotent of $S^{S I}$. Then the $\mathcal{H}$-class of $(X, e)$ is isomorphic to the subgroup of $H_{e}$ (the $\mathcal{H}$-class of $e$ ) taking $X$ to itself under left multiplication. In particular, if the maximal subgroups of $S$ are torsion-free, $S^{\mathrm{Sl}}$ is combinatorial (has only trivial subgroups).

Proof. One easily verifies that $(Y, t) \mathcal{H}(X, e)$ if and only if $Y=X, t \in H_{e}$ and $t X=X$. It now follows that the projection to $S$ gives the desired isomorphism. Since the $\mathcal{R}$-classes of $S^{S t}$ are finite, so are the $\mathcal{H}$-classes whence the final statement.

We remark that the $\mathcal{D}$-classes of $S^{\mathrm{SI}}$ are finite since the $\mathcal{L}$ and $\mathcal{R}$-classes are finite.

5.3. Applications We present an application to the theory of varieties. If $\mathbf{V}$ is a variety of inverse semigroups (a class closed under products, inverse subsemigroups, and quotients), then $\mathbf{S l ~} \mathbf{V} \mathbf{V}$ is the variety generated by inverse semigroups with an idempotent-pure morphism to an inverse semigroup in $\mathbf{V}$. A general nonsense-type argument shows if $F_{\mathbf{v}}(A)$ is the relatively free $A$-generated inverse semigroup in $\mathrm{V}$, then $F_{\mathrm{V}}(A)^{\mathbf{S I}}$ is the $A$-generated relatively free inverse semigroup in SL $\rightarrow$ V. Hence we can deduce the following.

THEOREM 5.13. Let $\mathbf{V}$ be a variety of inverse semigroups. Then the relatively free inverse semigroup in $\mathbf{S I}(m) \mathbf{V}$ on any finite set has finite $\mathcal{R}$-classes and Schützenberger 
graphs. Furthermore, it has a decidable word problem provided the corresponding free semigroup in $\mathbf{V}$ does and the finite members of $\mathbf{S I} ; \mathbf{V}$ are recursively enumerable.

PROOF. It is well known [6] that a residually finite, finitely generated algebra defined by a recursive set of relations has decidable word problem if its finite images are recursively enumerable. Under our hypotheses, this is the case for $F_{\mathrm{V}}(A)^{\mathrm{SI}}$.

COROLLARY 5.14. Suppose $\mathbf{V}$ is defined by a recursive set of equations of the form $u w^{-1}=u$, where $u=v w$, whose validity can be effectively tested in finite inverse semigroups; then $F_{\mathbf{v}}(A)$ has decidable word problem for any finite set $A$. In particular, the variety defined by the equation $t^{m}=t^{m+n}$ with $n \leq m$ has these properties.

ProOF. By Corollary 5.3, the variety $\mathbf{V}$ is fixed by the expansion (-) ${ }^{\mathrm{SI}}$ whence $F_{\mathbf{V}}(A)=F_{\mathbf{V}}(A)^{\mathbf{S I}}$ and hence is residually finite. The result now follows from the above theorem.

A similar argument to the above ones shows the following.

PROPOSITION 5.15. Let $S$ be an inverse semigroup with a recursive presentation over a finite set $A$ consisting of relations of the form $u w^{-1}=u$, where $u=v w$, which can effectively be checked in finite A-generated inverse semigroups. Then $S$ has a decidable word problem.

We mention that the expansion $S^{\mathbf{G p}}$ which relates to $\mathbf{G p}(\mathrm{m})$ (where $\mathbf{G p}$ is the variety of groups) in the same way that $S^{\text {St }}$ relates to $S \mathbf{S l}(\mathbf{V}$ was investigated in [13]. The reader is also referred to [17].

\section{A generalized prefix expansion}

In [3], Birget and Rhodes introduce an expansion of semigroups called the prefix expansion. If $S$ is a semigroup, then $S^{\mathrm{Pr}}$ consists of all pairs $(X, s)$ where $X$ is finite subset of $S^{1}$ containing 1 and $s$ and which is an $\leq_{\mathcal{R}}$-chain. The multiplication is given by $(X, s)(Y, t)=(X \cup s Y, s t)$. This semigroup is generated by the elements $(\{1, s\}, s)$ and two words in $S^{+}$map to the same element of $S^{\mathbf{P r}}$ if and only if they map to the same element of $S$ and their set of prefixes map to the same set of elements of $S$ (whence the name). Now if $G$ is a group, then every finite subset is an $\leq_{\mathcal{R}}$-chain and $G^{\mathbf{P r}}$ is an inverse semigroup where the inverse of $(X, g)$ is $\left(g^{-1} X, g^{-1}\right)$, and the projection $\eta_{G}: G^{\mathrm{Pr}} \rightarrow G$ is idempotent-pure. In fact, it is easy to see that $(X, g) \leq\left(X^{\prime}, g^{\prime}\right)$ if and only if $g=g^{\prime}$ and $X^{\prime} \subseteq X$. Thus, for each $g \in G$, the set $g \eta_{G}^{-1}$ has a maximum element, namely $(\{1, g\}, g)$, whence $G^{\mathrm{Pr}}$ is what is called an $F$-inverse monoid [10]. Our goal in this section is to generalize this expansion to inverse semigroups. A 
word of caution: for the remainder of this paper, if $S$ is an inverse semigroup, the notation $S^{\mathbf{P r}}$ will denote our new prefix expansion of inverse semigroups and not the Birget-Rhodes prefix expansion; in general the two only coincide on the class of groups.

6.1. Dual Prehomomorphisms and $F \cdot$ morphisms First we try to motivate why we wish to do this. In [22], Szendrei showed that $G^{\mathbf{P r}}$ is the universal $F$-inverse monoid with maximal group image $G$. That is, given any $F$-inverse monoid $I$ with maximal group homomorphism $\sigma: I \rightarrow G$, there is a unique homomorphism $\psi: G^{\mathrm{Pr}} \rightarrow I$ such that $\left(\max \left(g \eta_{G}^{-1}\right)\right) \psi=\max \left(g \sigma^{-1}\right)$.

The first author and Kellendonk [9] then showed that $G^{\mathbf{P r}}$ has another important universal property. To describe it, we must introduce some further concepts. The function $\eta_{G}^{*}: G \rightarrow G^{\mathrm{Pr}}$ given by $g \eta_{G}^{*}=\max \left(g \eta_{G}^{-1}\right)$ has the properties:

(1) $g_{1} \eta_{G}^{*} g_{2} \eta_{G}^{*} \leq g_{1} g_{2} \eta_{G}^{*}$

(2) $g^{-1} \eta_{G}^{*}=\left(g \eta_{G}^{*}\right)^{-1}$.

Such a function between inverse semigroups is called a dual prehomomorphism. As motivation for considering them, we mention partial actions of an inverse semigroup on a set, a concept we now introduce. Suppose $S$ is an inverse semigroup acting on a set $Y$ by partial bijections and $X \subseteq Y$. Then there is a partial action of $S$ on $X$ by restriction. The induced map from $S$ to $I(X)$ (the symmetric inverse monoid on $X)$ is an order-preserving dual prehomomorphism. This will be touched on again in Section 6.5.

It turns out that the map $\eta_{G}^{*}$ considered above is universal amongst all dual prehomomorphisms from $G$ to an inverse semigroup; that is, all dual prehomomorphisms $\varphi: G \rightarrow I$ factor through $\eta_{G}^{*}$ followed by a homomorphism. This result is due to Kellendonk and Lawson [9], developing an earlier result of Exel [7]. We shall shortly see that it follows from the result of Szendrei. Relationships between dual prehomomorphisms and $F$-inverse monoids can also be found in [16].

The following lemma is straightforward, but of frequent enough use to be worth isolating.

LEMMA 6.1. Let $\theta: S \rightarrow T$ be a dual prehomomorphism and $e \in E(S)$. Then $e \theta \in E(T)$.

Proof. We have the following: $e \theta=e \theta(e \theta)^{-1} e \theta=e \theta e \theta e \theta \leq e \theta e \theta \leq e \theta$.

Suppose $\varphi: S \rightarrow T$ is a surjective morphism of inverse semigroups such that $t \varphi^{-1}$ has a maximum element for each $t \in T$. Define $\varphi^{*}: T \rightarrow S$ by $t \varphi^{*}=\max t \varphi^{-1}$; then $\varphi^{*}$ is a dual prehomomorphism. We call $\varphi$ an $F$-morphism if $\varphi^{*}$ is order-preserving. Note that $S$ is an $F$-inverse monoid if and only if the natural surjection to its maximal 
group image is an $F$-morphism. One can check that a surjective morphism $\varphi$ is an $F$-morphism if and only if it has a right adjoint (viewed as a map of partially ordered sets). See [4] for more on homomorphisms of partially ordered semigroups which have right adjoints.

Observe that the assumption that $\varphi^{*}$ be order-preserving is not redundant. Indeed, let $S=\{x, y, x y\}$ be a free semilattice on $x$ and $y$ and $T=\{0,1\}$ (with multiplication). Define $\varphi: S \rightarrow T$ by $x \varphi=1, y \varphi=0$. Then $x=\max 1 \varphi^{-1}$ and $y=\max 0 \varphi^{-1}$, but $y=0 \varphi^{*} \not \leq x=1 \varphi^{*}$. Thus $\varphi$ is not an $F$-morphism.

We now aim to show that $F$-morphisms play the same role in the theory of idempotent-pure homomorphisms that $F$-inverse monoids play in the theory of $E$ unitary inverse semigroups; see [10, Chapter 7] for an exposition of this.

We mention that the $F$ in $F$-morphism stands for fermé meaning closed in French. This is because the order-preserving dual prehomomorphism $\varphi \varphi^{*}$ is a closure function on $S$. A closure function $\alpha: S \rightarrow S$ is an order-preserving dual prehomomorphism such that $s \leq s \alpha$ and $s \alpha=s \alpha \alpha$. Note that $\alpha$ is then a closure operator on $S$ (that is, an idempotent, increasing, order-preserving endomorphism of a partially ordered set). One can then prove the following straightforward lemma on closure functions.

LEMMA 6.2. Let $\alpha: S \rightarrow S$ be a closure function. Then

(1) $s \in E(S)$ if and only if $s \alpha \in E(S)$;

(2) $(a b) \alpha=(a \alpha b) \alpha=(a b \alpha) \alpha=(a \alpha b \alpha) \alpha$.

ProOF. If $s \in E(S)$, then $s \alpha \in E(S)$ by Lemma 6.1. If $s \alpha \in E(S)$, then $s \leq s \alpha$, so $s \in E(S)$. The second assertion is trivial.

COROLlaRY 6.3. Let $\varphi: S \rightarrow T$ be an F-morphism. Then $\varphi$ is idempotent-pure.

Proof. Suppose $s \eta$ is idempotent; then $s \eta \eta^{*}$ is an idempotent by Lemma 6.1. Since $\eta \eta^{*}$ is a closure function, $s \in E(S)$, as desired (using Lemma 6.2).

If $\alpha$ is a closure function, then the set of closed elements $S \alpha$ can be made into an inverse semigroup via the multiplication $s \cdot t=(s t) \alpha$. Moreover, $\alpha: S \rightarrow(S \alpha, \cdot)$ is an $F$-morphism with $\alpha \alpha^{*}=\alpha$. Conversely, if $\varphi: S \rightarrow T$ is an $F$-morphism, then $\left(S \varphi \varphi^{*}, \cdot\right)$ is isomorphic to $T$ and the $F$-morphisms commute with the isomorphisms. Thus the study of $F$-morphisms from $S$ is the same as the study of closure functions on $S$.

To highlight the importance of $F$-morphisms, we prove two factorization results for idempotent-pure surmorphisms of inverse semigroups. The first is a new result generalizing the fact that any $E$-unitary inverse semigroup embeds in an $F$-inverse monoid [10]; our proof, in fact, generalizes the proof of this older result. We then 
deduce, as a consequence, a result of Billhardt [1] on factoring idempotent-pure homomorphisms through $\lambda$-semidirect products.

As background, we recall some classical results and definitions about partially ordered semigroups.

A partially ordered semigroup is, of course, a semigroup equipped with a partial ordering which is compatible with the multiplication.

Let $S$ and $T$ be partially ordered semigroups. McFadden [14] defines a morphism $\theta: S \rightarrow T$ to be an $m$-homomorphism if for each $a \in S$ the set $a \theta \theta^{-1}$ has a maximum element $m_{a}$ such that $a \leq b$ implies $m_{a} \leq m_{b}$.

In what follows, every inverse semigroup is partially ordered with respect to its natural partial order. The following lemma is straightforward and we leave it to the reader.

LEMMA 6.4. Let $\theta: S \rightarrow T$ be a surjective homomorphism between inverse semigroups. Then $\theta$ is an $m$-homomorphism if and only if it is an F-morphism.

It follows that our notion of an ' $F$-morphism' is a special case of McFadden's notion of an ' $m$-homomorphism'.

O'Carroll [15, Theorem 3] proves the following result. Let $\theta: S \rightarrow T$ be a surjective order preserving homomorphism of partially ordered semigroups $S$ and $T$. Then $\theta$ can be factored $\theta=j \beta$ where $S \stackrel{j}{\rightarrow} M(S) \stackrel{\beta}{\rightarrow} T$ with $j$ an order preserving embedding, $\beta$ a surjective order preserving $m$-homomorphism, and $M(S)$ a partially ordered semigroup constructed from $S$ and $T$.

We would like to prove the analog of this result for inverse semigroups. However, in the case that $S$ and $T$ are both inverse, one finds that in general, $M(S)$ need not be inverse. Indeed, since injections and $F$-morphisms are idempotent-pure, only surjective idempotent-pure maps can have a factorization as an inclusion followed by an $F$-morphism.

Thus in seeking to formulate the analog of O'Carroll's result above, we must restrict our attention to surjective idempotent pure homomorphisms.

We need two lemmas. Recall that a subset $A$ of an inverse semigroup is said to be compatible if, for all $a, b \in A, a b^{-1}$ and $a^{-1} b$ are idempotents.

LEMMA 6.5. Let $\varphi: S \rightarrow T$ be a morphism of inverse semigroups. Then $\varphi$ is idempotent-pure if and only if $A \subseteq T$ compatible, implies $A \varphi^{-1}$ is compatible.

Proof. Suppose $\varphi$ is idempotent-pure and $A \subseteq T$ is compatible. Let $a, b \in A \varphi^{-1}$. Then $\left(a b^{-1}\right) \varphi=a \varphi(b \varphi)^{-1} \in E(T)$ since $A$ is compatible. Thus $a b^{-1} \in E(S)$ since $\varphi$ is idempotent-pure; dually $a^{-1} b \in E(S)$.

Conversely, suppose $\varphi^{-1}$ preserves compatibility and $e \in E(T)$. Then $e \varphi^{-1}$ is a compatible set. If $a \in e \varphi^{-1}$, then so are $a a^{-1}$ and $a^{-1}$. Hence $a=\left(a a^{-1}\right)\left(a^{-1}\right)^{-1} \in$ 
$E(S)$, as desired.

The following lemma is evident.

LemMa 6.6. Suppose $\varphi: S \rightarrow T$ is an $F$-morphism and $T^{\prime} \subseteq T$ is an inverse subsemigroup. Let $S^{\prime}=T^{\prime} \varphi^{-1}$ and let $\varphi^{\prime}: S^{\prime} \rightarrow T^{\prime}$ be the restriction. Then $\varphi^{\prime}$ is an F-morphism.

THEOREM 6.7. Let $\theta: S \rightarrow T$ be a surjective idempotent-pure homomorphism between inverse semigroups. Then there is an inverse semigroup $M(S)$, an embedding $j: S \rightarrow M(S)$, and an F-morphism $\beta: M(S) \rightarrow T$ such that $\theta=j \beta$.

PROOF. We use the Schein completion $C(S)$ of the inverse semigroup $S$ (see [10, 18]). The inverse semigroup $C(S)$ consists of all compatible order ideals of $S$. There is an embedding $\iota_{S}: S \rightarrow C(S)$ given by $s \mapsto[s]$, the principal order ideal generated by $s$. Similarly, we can consider $C(T)$ and $\iota(T)$. The reader should compare with the proof of [10, Proposition 7.1.4].

Define $\Theta: C(S) \rightarrow C(T)$ by $A \Theta=A \theta$. Since $\theta$ is surjective, $A \theta$ is a compatible order ideal. Clearly $\Theta$ is a homomorphism. Notice that $\theta \iota_{T}=\iota_{S} \Theta$, so $\Theta$ extends $\theta$. We show that $\Theta$ is an $F$-morphism. Let $B \in C(T)$. Then $B \theta^{-1} \in C(S)$ by Lemma 6.5. Since $B \theta^{-1} \Theta=B, \Theta$ is surjective. Moreover, we clearly have $B \theta^{-1}=\max B \Theta^{-1}$.

Let $M(S)=T \iota_{T} \Theta^{-1}$. Let us no longer distinguish $S \iota_{S}$ from $S$, and likewise $T \iota_{T}$ from $T$. Then $S \subseteq M(S)$ and the restriction $\Theta^{\prime}: M(S) \rightarrow T$ of $\Theta$ is an $F$-morphism by Lemma 6.6. Since $\Theta^{\prime}$ extends $\theta$, the result follows.

Note that Theorem 6.7, when restricted to the case where $T$ is a group, shows that each $E$-unitary inverse semigroup embeds in an $F$-inverse monoid.

As a corollary we obtain the following result of Billhardt [1].

COROLLARY 6.8. Let $\theta: S \rightarrow T$ be a surjective idempotent-pure homomorphism of inverse semigroups. Then there is a $\lambda$-semidirect product $E \rtimes^{\lambda} T$ with $E$ a semilattice, and an embedding $\iota: S \rightarrow E \rtimes^{\lambda} T$ such that $\theta=\imath \pi$ where $\pi$ is the semidirect product projection.

Proof. By Theorem 6.7, $\theta=j \beta$ with $j$ an embedding and $\beta: M(S) \rightarrow T$ an $F$-morphism. The congruence associated to $\beta$ is clearly a Billhardt congruence (see $[1,10]$ for the definition). Hence, by [10, Theorem 5.3.5], $\beta$ can be factored $i \pi$ where $i: M(S) \rightarrow K \operatorname{er} \beta^{T} \rtimes^{\lambda} T$ is an inclusion and $\pi$ is the semidirect product projection. Setting $\iota=j i$, we are done.

This result is also proved in [8] and can be deduced from the techniques of [19]. 
We thank the anonymous referee for pointing out that our Theorem 6.7 can, in turn, be deduced from Bilhardt's theorem and so the two results are formally equivalent.

We have thus far seen how we can associate an order-preserving dual prehomomorphism to any $F$-morphism. Now we complete the connection by going in the opposite direction.

PROPOSITION 6.9. Let $\psi: T \rightarrow S$ be an order-preserving dual prehomomorphism. Then there exists an inverse semigroup $I$, an $F$-morphism $\varphi: I \rightarrow T$, and a homomorphism $\rho: I \rightarrow S$ such that $\psi=\theta_{\varphi} \rho$.

Proof. Let $I=\{(s, t) \in S \times T \mid s \leq t \psi\}$. We claim $I$ is an inverse subsemigroup of $S \times T$. Indeed, if $\left(s_{1}, t_{1}\right),\left(s_{2}, t_{2}\right) \in I$, then

$$
t_{1} \psi t_{2} \psi \leq\left(t_{1} t_{2}\right) \psi
$$

so $s_{1} s_{2} \leq\left(t_{1} t_{2}\right) \psi$. Closure under inverse follows easily since $t^{-1} \psi=(t \psi)^{-1}$. The projection $\varphi$ to $T$ is an $F$-morphism since $(t \psi, t)=\max \left(t \varphi^{-1}\right)$ by definition of $I$, and if $t_{1} \leq t_{2} \in T$, then $t_{1} \psi \leq t_{2} \psi$ so $\left(t_{1} \psi, t_{1}\right) \leq\left(t_{2} \psi, t_{2}\right)$. Finally, if $\rho$ is the projection to $S$, then $\varphi^{*} \rho=\psi$.

One says that an $F$-morphism $\eta: U \rightarrow T$ is universal amongst $F$-morphisms to $T$ if whenever there is an $F$-morphism $\psi: S \rightarrow T$, there is a unique homomorphism $\alpha: U \rightarrow S$ with $\eta=\alpha \psi$ and $\eta^{*} \alpha=\psi^{*}$. If such an inverse semigroup $U$ exists, it must be unique up to isomorphism by the usual argument. One says that an order-preserving dual prehomomorphism $\theta: T \rightarrow U$ is universal amongst order-preserving dual prehomomorphisms from $T$ if, given any dual order-preserving prehomomorphism $\psi: T \rightarrow S$, there is a unique homomorphism $\rho: U \rightarrow S$ such that $\psi=\theta \rho$. Again, if such an inverse semigroup $U$ exists, it must be unique up to isomorphism.

THEOREM 6.10. Let $T$ be an inverse semigroup. Then if $\eta: U \rightarrow T$ is universal amongst $F$-morphisms to $T$, the associated dual prehomomorphism $\eta^{*}: T \rightarrow U$ is universal amongst order-preserving dual prehomomorphisms from $T$.

Proof. Let $\psi: T \rightarrow S$ be a dual prehomomorphism and let $I, \rho$, and $\varphi$ be as in the proof of Proposition 6.9. Then there exists a homomorphism $\alpha: U \rightarrow I$ such that $\alpha \varphi=\eta$ and $\eta^{*} \alpha=\varphi^{*}$. So $\psi=\varphi^{*} \rho=\eta^{*} \alpha \rho$. The uniqueness follows since if $\psi=\eta^{*} \beta$ with $\beta: U \rightarrow S$, then $\gamma=\beta \times \eta: U \rightarrow I$ is a morphism satisfying the properties demanded of $\alpha$. Hence $\gamma=\alpha$. But $\beta=\gamma \rho=\alpha \rho$.

Specializing to the case where $T$ is a group, we have by [22] that $\eta_{T}: T^{\mathrm{Pr}} \rightarrow T$ is universal amongst $F$-morphisms to $T$. Hence, Theorem 6.10 shows that $T^{\mathrm{Pr}}$ has the universal property proved by Kellendonk and Lawson [9]. That is, given any 
dual prehomomorphism $\theta: T \rightarrow S$ with $S$ an inverse semigroup, there is a unique homomorphism $\varphi: T^{\mathrm{Pr}} \rightarrow S$ such that $\eta_{T}^{*} \varphi=\theta$.

One might be tempted to prove a converse to Theorem 6.10. However, there is no need to do so. Once we show that universal $F$-morphisms exist, it will follow that universal dual prehomomorphisms exist and (by uniqueness) are constructed from $F$-morphisms in the above fashion.

6.2. $L$-representations Since $F$-morphisms are idempotent-pure (by Corollary 6.3), we can use the general theory of idempotent-pure homomorphisms to study them [10]. We begin by characterizing the $L$-representations of such inverse semigroups [10]. We briefly recall McAlister-O'Carroll triples. Let $S$ be an inverse semigroup, $X$ a partially ordered set, and $Y$ an order ideal of $X$ which is a semilattice in the induced order. Let $I(X, \leq)$ be the inverse monoid of order isomorphisms (acting on the left) between order ideals of $X$. If $P$ is a partially ordered set and $p \in P$, then $[p]$ will denote the order ideal generated by $p$. A McAlister- $O^{\prime}$ Carroll triple $(\gamma, q, Y)$ consists of a morphism $\gamma: S \rightarrow I(X, \leq)$ and a surjective morphism $q: Y \rightarrow E(S)$ such that:

(1) $(S \gamma) Y=X$.

(2) For each $y \in Y$ and $e \in E(S), y \in \operatorname{dom}(e \gamma)$ if and only if $y q \leq e$.

(3) For each $s \in S$, there exists $y \in Y$ such that $y q=s s^{-1}$ and $\left(s^{-1} \gamma\right)(y) \in Y$.

Usually, we denote $(s \gamma)(x)$ by $s x$. If $(\gamma, q, Y)$ is a McAlister-O'Carroll triple, then one obtains an inverse semigroup

$$
L(\gamma, q, Y)=\left\{(y, s) \in Y \times S \mid y q=s s^{-1}, s^{-1} y \in Y\right\}
$$

with product given by $(x, s)(y, t)=\left(s\left(s^{-1} x \wedge y\right), s t\right)$. The natural partial order turns out to be the product order. One can show that $E(L(\gamma, q, Y)) \cong Y$ and that the projection to $S$ is surjective and idempotent-pure. Conversely, see for instance [10], if $\varphi: T \rightarrow S$ is a surjective idempotent-pure morphism, then $T \cong L(\gamma, q, Y)$ for some McAlister-O'Carroll triple $(\gamma, q, Y)$ and under this isomorphism, $\varphi$ is transformed into the projection. One calls this an $L$-representation of $T$. Since $F$-morphisms are always idempotent-pure, we can attempt to characterize them in terms of their $L$ representations. In the case that $S$ is a group, and so one is considering an $F$-inverse monoid $T$, it is well known [10] that, in the $L$-representation of $T, X$ is a semilattice and $Y$ is a semilattice with maximum. Our characterization of $F$-morphisms will generalize this.

For $s \in S$, we set $s \mathbf{d}=s s^{-1}$ and $s \mathbf{r}=s^{-1} s$. It is shown in [10] that, given a McAlister-O'Carroll triple $(\gamma, q, Y)$, there is a unique order-preserving surjection $p: X \rightarrow E(S)$ extending $q$ such that:

(1) $\operatorname{dom}(e \gamma)=[e] p^{-1}$ for $e \in E(S)$. 
(2) If $x p=s \mathbf{r}$, then $(s x) p=s \mathbf{d}$.

From now on we supress $\gamma$ from the notation.

We shall need the following lemma about McAlister-O'Carroll triples.

LeMma 6.11. Suppose $(\gamma, q, Y)$ is a McAlister-O'Carroll triple and $x \in X$. Then there exist $y \in Y$ and $s \in S$ such that $y q=s \mathbf{r}$ and $s y=x$.

Proof. By assumption, $x=s y$ for some $y \in Y$ and $s \in S$. Thus $y \in \operatorname{dom}(s \mathbf{r})$ whence $y q \leq s \mathbf{r}$. Then $y \in \operatorname{dom}(y q)$ so $y q y=y$. Hence $x=s y=s(y q) y$ and $(s(y q)) \mathbf{r}=y q s \mathbf{r}(y q)=y q$. So $s(y q)$ is as desired.

PROPOSITION 6.12. Let $(\gamma, q, Y)$ be a McAlister-O'Carroll triple and $p$ be as above. Then the projection $\varphi: L(\gamma, q, Y) \rightarrow S$ is an $F$-morphism if and only if for each $e \in E(S)$, pairs of elements of $e p^{-1}$ have a meet, $e q^{-1}$ has a maximum element, and the function $e \mapsto \max \left(e q^{-1}\right)$ is order-preserving.

ProOF. Suppose that the latter condition holds. We show that $\varphi$ is an $F$-morphism. For $e \in E(S)$, let $y_{e}=\max \left(e q^{-1}\right)$ and let $s \in S$. Then $\left(s y_{s \mathrm{r}}\right) p=s \mathbf{d}$ so

$$
y_{s \mathbf{d}} \wedge s y_{s \mathbf{r}} \in s \mathbf{d} q^{-1}
$$

(recall: $Y$ is an order ideal). Also

$$
s^{-1}\left(y_{s \mathbf{d}} \wedge s y_{s \mathbf{r}}\right) \leq s^{-1} s y_{s \mathbf{r}}=y_{s \mathbf{r}}
$$

and hence is in $Y$. Thus $\left(y_{s d} \wedge s y_{s r}, s\right) \in L(\gamma, q, Y)$. Suppose $(y, s) \in L(\gamma, q, Y)$. We claim that $y \leq y_{s \mathbf{d}} \wedge s y_{s \mathrm{r}}$. Indeed, $y q=s \mathbf{d}$ so $y \leq y_{s \mathrm{~d}}$. But $s^{-1} y \in s \mathbf{r} q^{-1}$ so $s^{-1} y \leq y_{s \mathrm{r}}$ whence $y \leq s y_{s \mathrm{r}}$. It follows that $\left(y_{s \mathrm{~d}} \wedge s y_{s \mathrm{r}}, s\right)$ is the maximum element of $s \varphi^{-1}$. Suppose $s_{1} \leq s_{2}$, then $s_{1} \mathbf{d} \leq s_{2} \mathbf{d}$ and $s_{1} \mathbf{r} \leq s_{2} \mathbf{r}$, so $y_{s_{1} \mathbf{d}} \leq y_{s_{2} \mathbf{d}}$ and similarly $y_{s_{1} \mathrm{r}} \leq y_{s_{2} \mathrm{r}}$. Thus

$$
s_{1} y_{s_{1} \mathbf{r}}=s_{2} y_{s_{1} \mathbf{r}} \leq s_{2} y_{s_{2} \mathbf{r}}
$$

so $\left(y_{s_{\mathbf{d}} \mathrm{d}} \wedge s_{1} y_{s_{1} \mathbf{r}}, s_{1}\right) \leq\left(y_{s_{2} \mathrm{~d}} \wedge s_{2} y_{s_{2} \mathrm{r}}, s_{2}\right)$. Hence the projection is an $F$-morphism.

Suppose that the projection $\varphi: L(\gamma, q, Y) \rightarrow S$ is an $F$-morphism. To show that pairs of elements of $e p^{-1}$ have a meet, it suffices to show that, for $x \in e p^{-1}$ and $y \in e q^{-1}$, there exists $x \wedge y$. Indeed, suppose we have shown this and that $x, x^{\prime} \in e p^{-1}$. Then, by Lemma $6.11, x^{\prime}=s z$ for some $z \in Y$ and $s \in S$ such that $z q=s \mathbf{r}$. So $e=x^{\prime} p=s \mathbf{d}$ whence $s^{-1} x$ exists and $\left(s^{-1} x\right) p=s \mathbf{r}$. Therefore $z \wedge s^{-1} x$ and $s\left(z \wedge s^{-1} x\right)$ exist. But it is then straightforward to verify that $x \wedge x^{\prime}=s\left(z \wedge s^{-1} x\right)$.

Suppose $x \in e p^{-1}$ and $y \in e q^{-1}$. Let $x=s z$ with $z \in Y$ and $z q=s \mathbf{r}$. Then $e=(s z) p=s \mathbf{d}$. Let $(k, s)=\max \left(s \varphi^{-1}\right)$. Then $k q=s \mathbf{d}$ so $y \wedge k$ exists. But $(y \wedge k) q=s \mathbf{d}$, so $s^{-1}(y \wedge k)$ exists. Also, since $s^{-1} k \in Y$ and $Y$ is an order ideal, 
$s^{-1}(y \wedge k) \in Y$. Thus we can take $s^{-1}(y \wedge k) \wedge z$ which is in $Y$. It is then straightforward to check that $q$ of this expression is $s \mathbf{r}$. So let $h=s\left(s^{-1}(y \wedge k) \wedge z\right)$. We claim $h=x \wedge y$. Indeed, $h \leq s z=x$. Also $h \leq s s^{-1} y=y$. Suppose that $j \leq x, y$. Then $j p \leq e=s \mathbf{d}$ so $s^{-1} j \leq s^{-1} x=z$. Hence $j, s^{-1} j \in Y$ and $j q \leq e=s \mathbf{d}$. So $(j, j q s) \in L(\gamma, q, Y)$. Suppose $\left(k^{\prime}, j q s\right)=\max \left((j q s) \varphi^{-1}\right)$. Then $k^{\prime} \leq k$ and $j \leq k^{\prime}$, so $j \leq k$. Putting this all together, we see that $s^{-1} j \leq s^{-1}(y \wedge k) \wedge z$ so $j \leq h$.

We now show that $e q^{-1}$ has a maximum. Let $(y, e)=\max \left(e \varphi^{-1}\right)$. Suppose $y^{\prime} \in e q^{-1}$. Then $\left(y^{\prime}, e\right) \in L(\gamma, q, Y)$ so $y^{\prime} \leq y$. It follows immediately that $e \mapsto \max \left(e q^{-1}\right)$ preserves order since $e \mapsto \max \left(e \varphi^{-1}\right)$ preserves order.

6.3. The expansion For each inverse semigroup $S$, we shall construct an inverse semigroup $S^{\mathrm{Pr}}$ together with an $F$-morphism $\eta_{S}: S^{\mathrm{Pr}} \rightarrow S$ which will be the universal $F$-morphism to $S$. The construction $S \mapsto S^{\mathbf{P r}}$ is functorial and the surjective morphisms $\eta_{S}$ for the components of a natural transformation from (_) $)^{\mathbf{P r}}$ to the identity functor. It follows that $\left({ }_{-}\right)^{\mathbf{P r}}$ is an expansion of inverse semigroups.

We shall construct $S^{\mathbf{P r}}$ by first constructing a McAlister-O'Carroll triple from $S$ which satisfies Proposition 6.12 of Section 6.2. This will enable us to build $S^{\mathbf{P r}}$ and $\eta_{s}$ explicitly. We shall then prove that $\eta_{s}$ is the required universal $F$-morphism. The triple $\left(\gamma_{s}, q_{s}, Y_{S}\right)$ will be constructed in several stages with various intermediary propositions which prove that things are well defined.

This expansion will be similar to $S^{\mathrm{Sl}}$, only we will take $S$ as the generating set and we shall be interested only in vertices of Schützenberger graphs rather than subgraphs.

For those readers preferring a more direct construction, the reader is referred to Proposition 6.16.

If $A$ is a set, we use $\mathcal{P}_{\text {fin }}(A)$ for the collection of non-empty finite subsets of $A$. Let

$$
X_{S}=\left\{A \mid A \in \mathcal{P}_{\text {fin }}\left(R_{e}\right) \text { for some } e \in E(S)\right\}
$$

and

$$
Y_{S}=\left\{A \in X_{S} \mid A \cap E(S) \neq \emptyset\right\} .
$$

Suppose $A \in \mathcal{P}_{\text {fin }}\left(R_{e}\right)$ and $B \in \mathcal{P}_{\text {fin }}\left(R_{f}\right)$. Define $A \wedge B=f A \cup e B$. It is easy to see that $A \wedge B \in \mathcal{P}_{\text {fin }}\left(R_{e f}\right)$. Also note that $A \wedge A=e A \cup e A=A$ and $A \wedge B=B \wedge A$. So $X_{S}$ is a semilattice.

Proposition 6.13. Let $A \in \mathcal{P}_{\mathrm{fin}}\left(R_{e}\right)$ and $B \in \mathcal{P}_{\mathrm{fin}}\left(R_{f}\right)$. Then $A \leq B$ if and only if $e \leq f$ and $e B \subseteq A$.

Proof. Suppose $A \leq B$. Then $A=A \wedge B=f A \cup e B \in \mathcal{P}_{\text {fin }}\left(R_{e f}\right)$ so $e=e f$ and $e B \subseteq A$. For the converse, $A \wedge B=f A \cup e B=A \cup e B=A$.

It is now easy to see that $Y_{S}$ is an order ideal (and hence a semilattice in its induced order). Define $\gamma_{S}: S \rightarrow I\left(X_{S}, \leq\right)$ as follows: 
(1) $\operatorname{dom}\left(s \gamma_{S}\right)=\left\{A \in X_{S} \mid A \in \mathcal{P}_{\text {fin }}\left(R_{e}\right), e \leq s \mathbf{r}\right\}$;

(2) $s \gamma(A)=\{s a \mid a \in A\}$.

Again, we supress $\gamma$ and merely write $s A$. This action is, in some sense, induced by the Preston-Wagner representation of $S$ and the usual left action of $S$ on its power set. If $S$ is a semigroup, $s \in S$, and $X \subseteq S$, then we will also let $s X=\{s x \mid x \in X\}$. There should be no confusion since the above actions differ only in their domain of definition.

PROPOSITION 6.14. The function $\gamma_{S}$ is a well-defined homomorphism.

Proof. First note that $\operatorname{dom}\left(s \gamma_{S}\right)$ is an order ideal. Also, it is easy to see that

$$
\operatorname{ran}\left(s \gamma_{S}\right)=\left\{A \in X_{S} \mid A \in \mathcal{P}_{\text {fin }}\left(R_{e}\right), e \leq s \mathbf{d}\right\}
$$

Suppose $A \leq B \in \operatorname{dom}\left(s \gamma_{S}\right), A \in \mathcal{P}_{\text {fin }}\left(R_{e}\right), B \in \mathcal{P}_{\text {fin }}\left(R_{f}\right)$, and $e, f \leq s \mathbf{r}$. Then $e \leq f$ and $e B \subseteq A$; so $\operatorname{ses}^{-1} \leq s f s^{-1}$. Now $\operatorname{ses}^{-1} s B=s e B \subseteq s A$. Thus $s B \leq s A$. We note that $s^{-1} \gamma_{s}$ is the inverse morphism to $s \gamma_{s}$ and so $s \gamma$ is an isomorphism of order ideals of $X$.

Now $\operatorname{dom}\left((s t) \gamma_{s}\right)=\left\{A \in X_{S} \mid A \in \mathcal{P}_{\text {fin }}\left(R_{e}\right), e \leq t^{-1} s \mathbf{r} t\right\}$. However, for $A \in$ $\operatorname{dom}\left(t \gamma_{s}\right), t A \in \operatorname{dom}\left(s \gamma_{S}\right)$ if and only if $A \in \mathcal{P}_{\text {fin }}\left(R_{e}\right)$ with $e \leq t^{-1} t$ and $t e t^{-1} \leq s^{-1} s$ which is if and only if $e=t^{-1} t e t^{-1} t \leq t^{-1} s \mathbf{r} t$. In this case $(s t) A=s(t A)$.

Define $q_{S}: Y_{S} \rightarrow E(S)$ by letting, for $A \in \mathcal{P}_{\text {fin }}\left(R_{e}\right), A q_{S}=e$. Then $q_{s}$ is a surjective homomorphism. Suppose $A \in \mathcal{P}_{\text {fin }}\left(R_{e}\right)$ and let $s \in A$. Then $s \mathbf{d}=e$ and $s^{-1} A \in Y_{S}$. If $A \in Y_{S}$ and $e \in E(S)$, then $A \in \operatorname{dom}\left(e \gamma_{S}\right)$ if and only if $A q \leq e$. Finally, if $s \in S$, then $R_{s \mathrm{~d}} \in Y_{S}, R_{s \mathrm{~d}} q_{s}=s s^{-1}$ and $s^{-1} R_{s \mathrm{~d}} \in Y_{S}$.

We have thus proved the following.

THEOREM 6.15. $\left(\gamma_{s}, q_{s}, Y_{S}\right)$ is a McAlister-O'Carroll triple.

Note that if $A \in \mathcal{P}_{\text {fin }}\left(R_{e}\right)$, then $A p_{S}=e$ where $p_{S}$ is the extension of $q_{S}$ to $X_{S}$.

We let $S^{\mathrm{Pr}}=L\left(\gamma_{S}, q_{S}, Y_{S}\right)$ and $\eta_{S}: S^{\mathrm{Pr}} \rightarrow S$ be the projection. Then, by Proposition 6.12, $\eta_{S}$ is an $F$-morphism. Indeed, since $X_{S}$ is a semilattice, we just need to show that $e q_{S}^{-1}$ has a maximum element for each $e \in E(S)$ and that the function $e \mapsto \max \left(e q_{S}^{-1}\right)$ preserves order. But clearly $\{e\}$ is such a maximum element and if $e \leq f$, then $\{e\} \leq\{f\}$. Finally, note that if $\psi: S \rightarrow T$ is a homomorphism of inverse semigroups, then there is an induced morphism $\psi^{*}: S^{\mathbf{P r}} \rightarrow T^{\mathbf{P r}}$ sending $(A, s)$ to $(A \psi, s \psi)$. Clearly $\eta$ is a natural transformation to the identity functor.

We now wish to give a description of $S^{\mathrm{Pr}}$ in terms of the operation of union on $\mathcal{P}_{\text {fin }}(S)$ and the usual left action of $S$ on this set. 
PROPOSITION 6.16. $S^{\mathrm{Pr}}=\left\{(A, s) \in X_{S} \times S \mid s \mathrm{~d}, s \in A\right\}$ with multiplication:

$$
(A, s)(B, t)=\left(s t t^{-1} s^{-1} A \cup s B, s t\right) \text {. }
$$

Proof. If $(A, s) \in S^{\mathbf{P r}}$, then $A \in Y_{S}$ and $A q_{s}=s \mathrm{~d}$, whence $s \mathbf{d} \in A$. Also, since $s^{-1} A \in Y_{S}, s^{-1} s \in s^{-1} A$ and so $s \in A$. Conversely, if $s \mathrm{~d}, s \in A$, then $A, s^{-1} A \in Y_{S}$ and $A q=s \mathrm{~d}$. So $(A, s) \in S^{\mathrm{Pr}}$. As for the multiplication,

$$
(A, s)(B, t)=\left(s\left(s^{-1} A \wedge B\right), s t\right)
$$

by definition of multiplication in an $L$-semigroup, but

$$
s^{-1} A \wedge B=t t^{-1} s^{-1} A \cup s^{-1} s B,
$$

so $s\left(s^{-1} A \wedge B\right)=s t t^{-1} s^{-1} A \cup s B$. The result follows.

If $S$ is a group, then $X_{S}=\mathcal{P}_{\text {fin }}(S)$ and the above result shows that $S^{\mathrm{Pr}}$ is the usual prefix expansion of $S$. Note that if $S$ is finite, then $X_{S}$ is finite whence $S^{\mathrm{Pr}}$ is also finite.

It is easy to see that $S^{\mathrm{Pr}}$ is a subsemigroup of the $\lambda$-semidirect product $\mathcal{P}_{\text {fin }}(S) \rtimes^{\lambda} S$. All that remains is to show that $\eta_{S}$ is universal.

THEOREM 6.17. Let $S$ be an inverse semigroup. Then the morphism $\eta_{s}: S^{\mathrm{Pr}} \rightarrow S$ is universal amongst $F$-morphisms to $S$.

ProOF. Let $(\gamma, q, Y)$ be a McAlister-O'Carroll triple as in Proposition 6.12. Let $p$ be the extension of $q$ to $X$. For $e \in E(S)$, we let $y_{e}=\max \left(e q^{-1}\right)$. Since morphisms in the comma category of idempotent-pure morphisms over $S$ correspond to morphisms (in the obvious sense) of McAlister-O'Carroll triples, we just need to show that there is a unique morphism $\psi: X_{S} \rightarrow X$ preserving the structure of a McAlister-O'Carroll triple, satisfying

$$
\{s \mathbf{d}, s\} \psi=y_{s \mathbf{d}} \wedge s y_{s \mathbf{r}} .
$$

The induced morphism $\bar{\psi}: S^{\mathrm{Pr}} \rightarrow L(\gamma, q, Y)$ defined by $\psi \times 1_{S}$ will then be the unique morphism preserving maxima.

Let $A \in X, A p_{s}=e$. We define $\psi$ by $A \psi=\bigwedge_{s \in A} s y_{s r}$. Since $s \in A, s \mathbf{d}=e$ and so $\left(s y_{s \mathrm{r}}\right) p=s \mathbf{d}$. Thus, by hypothesis on $(\gamma, q, Y), A \psi$ is well defined. Also, $\psi q=q_{s}$ (obvious) and $Y_{S} \psi \subseteq Y$ (since, for $A \in Y_{S}, y q \in A$, so $A \psi \leq y q y_{y q}=y_{y q}$ ). Clearly, $\{s \mathbf{d}, s\} \psi=y_{s \mathbf{d}} \wedge s y_{s \mathrm{r}}$.

To see that $\psi$ preserves order, suppose that $A \leq B$ with $A \in \mathcal{P}_{\text {fin }}\left(R_{e}\right), B \in \mathcal{P}_{\text {fin }}\left(R_{f}\right)$. Then $e \leq f$ and $e B \subseteq A$. Observe that, for $t \in B$, $(e t) \mathbf{r}=t^{-1} e t \leq t^{-1} t=t \mathrm{r}$, so $y_{(e t) \mathrm{r}} \leq y_{t \mathrm{r}}$ whence $(e t) y_{(e t) \mathrm{r}}=t y_{(e t) \mathrm{r}} \leq t y_{\mathrm{tr}}$. Therefore,

$$
A \psi \leq \bigwedge_{t \in B}(e t) y_{(e t) \mathrm{r}} \leq B \psi
$$


Now we show that $\left.\psi\right|_{Y_{S}}$ preserves meets. Let $A, B \in Y_{S}$. Since $\psi$ preserves order, we need only show $A \psi \wedge B \psi \leq(A \wedge B) \psi$. Suppose $A q_{s}=e, B q_{s}=f$. Now

$$
(A \wedge B) \psi=(f A \cup e B) \psi=\bigwedge_{s \in A} f s y_{(f s) \mathbf{r}} \wedge \bigwedge_{t \in B} e t y_{(e t) \mathbf{r}}
$$

On the other hand,

$$
A \psi \wedge B \psi=\bigwedge_{s \in A} s y_{s \mathrm{r}} \wedge \bigwedge_{t \in B} t y_{t \mathrm{r}}=\bigwedge_{s \in A}\left(s y_{s \mathrm{r}} \wedge y_{f}\right) \wedge \bigwedge_{t \in B}\left(t y_{t \mathrm{r}} \wedge y_{e}\right)
$$

where the last equality follows because $e \in A, f \in B$. Thus, it suffices to show that, for $s \in A, s y_{s \mathbf{r}} \wedge y_{f} \leq f s y_{(f s) \mathrm{r}}$ and dually for $t \in B$.

Since $Y$ is an ideal, $s y_{s r} \wedge y_{f} \in Y$. Also $\left(s y_{s r}\right) p=e$, so $\left(s y_{s \mathrm{r}} \wedge y_{f}\right) q \leq e f$ whence $s y_{s \mathbf{r}} \wedge y_{f} \leq y_{e f}$. Since $y_{e f} \leq y_{f}$,

$$
s y_{s \mathbf{r}} \wedge y_{f}=s y_{s \mathbf{r}} \wedge y_{f} \wedge y_{e f}=s y_{s \mathbf{r}} \wedge y_{e f} .
$$

Now observe $s^{-1}\left(s y_{s \mathbf{r}} \wedge y_{e f}\right) \leq y_{s \mathbf{r}} \wedge s^{-1} y_{e f}$. However, the latter is $y_{s \mathrm{r}} \wedge s^{-1} f y_{e f}$. But

$$
\left(y_{s \mathbf{r}} \wedge s^{-1} f y_{e f}\right) q \leq s \mathbf{r}\left(s^{-1} f\right) \mathbf{d}=s^{-1} f s=(f s) \mathbf{r},
$$

so $y_{s \mathbf{r}} \wedge s^{-1} y_{e f} \leq y_{(f s) \mathbf{r}}$. We conclude $s^{-1}\left(s y_{s \mathbf{r}} \wedge y_{f}\right) \leq y_{(f s) \mathbf{r}}$ whence $s y_{s \mathbf{r}} \wedge y_{f} \leq$ $s y_{(f s) \mathrm{r}}=f s y_{(f s) \mathrm{r}}$, as desired.

Finally, we show that $\psi$ preserves the action. Suppose that $t \in S$ and $A p \leq t \mathbf{r}$. Then, for $s \in A,(t s) \mathbf{r}=s^{-1} t^{-1} t s \leq s^{-1} s$ and $s^{-1} s=s^{-1} s s^{-1} s \leq s^{-1} t^{-1} t s=(t s) \mathbf{r}$ (since $s s^{-1} \leq t^{-1} t$ ) whence $(t s) \mathbf{r}=s \mathbf{r}$. So

$$
(t A) \psi=\bigwedge_{s \in t A} s y_{s r}=\bigwedge_{s \in A} t s y_{(t s) \mathrm{r}}=\bigwedge_{s \in A} t s y_{s \mathrm{r}} .
$$

But it is easy to check that multiplication by $t$ preserves finite meets (since it is an order isomorphism with inverse, multiplication by $\left.t^{-1}\right)$ so the right hand side is $t(A \psi)$.

To show that $\psi$ is unique, suppose $\tau: X_{S} \rightarrow X$ induces another morphism of McAlister-O'Carroll triples satisfying (*). First of all, since, for all $A \in X_{S}, A=s B$ for some $s \in S, B \in Y_{S}$, and since $\tau$ preserves the action, it suffices to show that $\tau$ agrees with $\psi$ on $Y_{S}$. Let $A=\left\{e, s_{1}, \ldots, s_{n}\right\} \in Y_{S}$ with $A q_{S}=e$. Then

$$
A=\left\{e, s_{1}\right\} \cup \cdots \cup\left\{e, s_{n}\right\}=\left\{e, s_{1}\right\} \wedge \cdots \wedge\left\{e, s_{n}\right\} .
$$

Since, for all $i,\left\{e, s_{i}\right\} \tau=y_{e} \wedge s_{i} y_{s_{i} \mathbf{r}}$ by $(*)$, and since $\left.\tau\right|_{Y_{s}}$ preserves meets, it follow that $A \tau=\bigwedge_{s \in A}\left(y_{e} \wedge s y_{s r}\right)=A \psi($ since $e \in A)$. 
6.4. Structure We now study the structure of $S^{\mathrm{Pr}}$. The following results are straightforward deductions from the structure of $L$-semigroups.

PROPOSITION 6.18. Let $S$ be an inverse semigroup.

(1) $(A, s) \leq(B, t)$ if and only if $s \leq t$ and $s s^{-1} B \subseteq A$.

(2) $(A, s) \mathcal{R}(B, t)$ if and only if $A=B$.

(3) $(A, s) \mathcal{L}(B, t)$ if and only if $s^{-1} A=t^{-1} B$.

(4) Let $(A, e) \in E\left(S^{\mathrm{Pr}}\right)$ and $H_{e}^{\prime}=\left\{s \in H_{e} \mid s A=A\right\}$. Then $\eta_{s}$ induces an isomorphism of $H_{(A, e)}$ with $H_{e}^{\prime}$.

(5) $(A, s) \mathcal{D}(B, t)$ if and only if there exists $u \in S$ such that $u \mathbf{d}=s \mathbf{r}, u \in s^{-1} A$, and $u^{-1} s^{-1} A=B$.

COROLlARY 6.19. The $\mathcal{R}$-classes of $S^{\mathrm{Pr}}$ are finite; hence $S^{\mathrm{Pr}}$ is residually finite. Moreover, if the maximal subgroups of $S$ are torsion-free, then $S^{\mathrm{Pr}}$ is combinatorial.

PROOF. The first assertion follow from Proposition 6.18 (2) since there are only finitely many elements of $S^{\mathrm{Pr}}$ of the form $(A, s)$ (since $A$ is finite). The last assertion follows since the $\mathcal{H}$-classes of $S^{\operatorname{Pr}}$ must then be finite and, by Proposition 6.18 (4), are isomorphic to subgroups of the maximal subgroups of $S$.

We end this subsection by observing that $S^{\mathrm{Pr}}$ is generated by $S$ via the map

$$
s \mapsto(\{s \mathbf{d}, s\}, s) .
$$

Indeed, this follows from the universal property since if $T \subseteq S^{\operatorname{Pr}}$ is the inverse subsemigroup generated by the elements of the form $(\{s \mathrm{~d}, s\}, s)$, then the restriction $\left.\eta_{S}\right|_{T}: T \rightarrow S$ would be an $F$-morphism and would be universal. Since the projection from $S^{\mathbf{P r}}$ is idempotent-pure, there is a natural surjective morphism $\psi: S^{\mathrm{SI}} \rightarrow S^{\mathrm{Pr}}$ (where we view $S$ as generated by $S$ ). This allows an alternate proof that if $S$ is finite, then so is $S^{\mathbf{P r}}$. This map is easily seen to take a pair $(X, s)$ to the pair $(\operatorname{Ver}(X), s)$ (defined earlier).

6.5. Applications Our main application is to partial actions of inverse semigroups. A partial action of an inverse semigroup $S$ on a set $X$ consists of an order-preserving dual prehomomorphism $\theta: S \rightarrow I(X)$. These most commonly arise from a restricting an action of $S$ by partial bijections of a set $Y$ to a subset $X \subseteq Y$. We say the partial action is graded if there is a map $p: X \rightarrow E(S)$ such that: for each idempotent $e \in E(S)$, there exists $e x$ if and only if $x p \leq e$; if $x p=s^{-1} s$, then $(s x) p=s s^{-1}$. The first and third author can show that all graded partial actions arise from restricting actions to a subset; that is, extending the terminology of [9], graded partial actions can be globalized. It is not apparent this is true of all partial actions of inverse semigroups. 
For applications of partial group actions, see Exel [7] and the first author and Kellendonk [9].

PROPOSITION 6.20. Let $S$ be an inverse semigroup and $X$ a set. Then all partial actions of $S$ on $X$ are of the form $\eta_{S}^{*} \varphi$ where $\varphi: S^{\mathrm{Pr}} \rightarrow I(X)$ is an action.

PROOF. By the universal property of $S^{\mathrm{Pr}}$ and by Theorem 6.10 , there exists a bijection between homomorphisms $\varphi: S^{\mathrm{Pr}} \rightarrow I(X)$ and order-preserving dual prehomomorphisms $\theta: S \rightarrow I(X)$ given by $\varphi \mapsto \eta_{S}^{*} \varphi$.

\section{Acknowledgements}

The second author was partially funded by the Excellency Center 'Group Theoretic Methods in the study of Algebraic Varieties' of the Israel Science foundation, INTAS grant 99-1224, Binational Science Foundation of Israel and the NSF. The third author was supported in part by NSF-NATO postdoctoral fellowship DGE-9972697, Praxis XXI scholarship BPD 16306 98, by Project SAPIENS 32817/99, by INTAS grant 991224, and by FCT through Centro de Matemática da Universidade do Porto. Much of this research took place at the Quasicrystals Workshop at the University of Essex funded by EPSRC grant number GR/M6544.

\section{References}

[1] B. Billhardt, 'On a wreath product embedding and idempotent pure congruences of inverse semigroups', Semigroup Forum 45 (1992), 45-54.

[2] J. C. Birget and J. Rhodes, 'Almost finite expansions of arbitrary semigroups', J. Pure Appl. Algebra 32 (1984), 239-287.

[3] _ ' Group theory via global semigroup theory', J. Algebra 120 (1989), 284-300.

[4] T. S. Blyth and M. F. Janowitz, Residuation theory, International Series of Monographs in Pure and Applied Mathematics 102 (Pergamon Press, Oxford, 1972).

[5] T. C. Brown, 'An interesting combinatorial method in the theory of locally finite semigroups', Pacific J. Math. 36 (1971), 285-289.

[6] T. Evans, 'Some connections between residual finiteness, finite embeddability and the word problem', J. London Math. Soc. 1 (1969), 399-403.

[7] R. Exel, 'Partial actions of groups and actions of inverse semigroups', Proc. Amer. Math. Soc. 126 (1998), 3481-3494.

[8] G. M. S. Gomes and M. B. Szendrei, 'Idempotent pure extensions by inverse semigroups via quivers', J. Pure Appl. Algebra 127 (1998), 15-38.

[9] J. Kellendonk and M. V. Lawson, 'Partial actions of groups', Internat. J. Algebra Comput. 14 (2004), 87-114.

[10] M. V. Lawson, Inverse Semigroups: The theory of partial symmetries (World Scientific, Singapore, 1998). 
[11] S. W. Margolis and J. C. Meakin, 'E-unitary inverse monoids and the Cayley graph of a group presentation', J. Pure Appl. Algebra 58 (1989), 45-76.

[12] - 'Inverse monoids, trees, and context-free languages', Trans. Amer. Math. Soc. 335 (1993), 259-276.

[13] S. W. Margolis, J. C. Meakin and J. B. Stephen, 'Free objects in certain varieties of inverse semigroups', Canad. J. Math. 42 (1990), 1084-1097.

[14] R. McFadden, 'On homomorphisms of partially ordered semigroups', Acta Sci. Math. Szeged 28 (1967), 241-249.

[15] L. O'Carroll, 'A class of congruences on a posemigroup', Semigroup Forum 3 (1971), 652-666.

[16] M. Petrich, Inverse semigroups (Wiley, New York, 1984).

[17] M. Petrich and N. Reilly, 'A network of congruences on an inverse semigroup', Trans. Amer. Math. Soc. 270 (1982), 309-325.

[18] B. M. Schein, 'Completions, translational hulls, and ideal extensions of inverse semigroups', Czechoslovak Math. J. 23 (1973), 575-610.

[19] B. Steinberg, 'Factorization theorems for morphisms of ordered groupoids and inverse semigroups', Proc. Edinburgh Math. Soc. 44 (2001), 549-569.

[20] _ - 'Finite state automata: A geometric approach', Trans. Amer. Math. Soc. 353 (2001), 3409-3464.

[21] J. B. Stephen, 'Presentations of inverse monoids', J. Pure Appl. Algebra 63 (1990), 81-112.

[22] M. B. Szendrei, 'A note on Birget-Rhodes expansion of groups', J. Pure Appl. Algebra 58 (1989), 93-99.

Division of Mathematics

School of Informatics

University of Wales

Gwynedd LL57 1UT

Wales

e-mail: m.v.lawson@bangor.ac.uk
Department of Mathematics

Bar Ilan University

52900 Ramat Gan

Israel

e-mail: margolis@macs.biu.ac.il

\author{
Department of Pure Mathematics \\ Faculty of Sciences \\ University of Porto \\ 4099-002 Porto \\ Portugal \\ Current Address: \\ School of Mathematics and Statistics \\ Carleton University \\ 1125 Colonel By Drive \\ Ottawa ON, K1S 5B6 \\ Canada \\ e-mail: bsteinbg@math.carleton.ca
}

